\title{
Menschenrechtsbildung aus psychologischer Perspektive
}

\author{
Berit Ötsch
}

\begin{abstract}
Zusammenfassung: Menschenrechtsbildung soll Menschen dazu befähigen, Menschenrechte zu verstehen und sich für die eigenen sowie die Rechte anderer einzusetzen, sodass langfristig eine Kultur der Menschenrechte entstehen kann. Sie wird als umfassende Aufgabe verstanden, die neben der Vermittlung von Wissen ebenso die Auseinandersetzung mit Werten und Einstellungen stimulieren und Handlungskompetenz fördern soll, was als Lernen über, durch und für die Menschenrechte bezeichnet wird. In dem Artikel wird aus psychologischer Perspektive untersucht, inwieweit Menschenrechtsbildung als dreidimensionale Aufgabe der Förderung von Wissen, Werten und Kompetenzen umsetzbar ist und wo sie diesbezüglich an ihre Grenzen stößt, auch im Hinblick auf die Frage nach der Realisierbarkeit der angestrebten Menschenrechtskultur. Die hohen Erwartungen an die Menschenrechtsbildung werden mit dem Ziel hinterfragt, ein realistischeres Bild dessen zu zeichnen, was die Menschenrechtsbildung an Ergebnissen tatsächlich erbringen könnte.
\end{abstract}

Schlüsselwörter: Menschenrechtsbildung $\cdot$ Psychologie $\cdot$ Kognitive Entwicklung · Kompetenzen · Werteerziehung

\section{Human rights education from a psychological point of view}

\begin{abstract}
Human rights education is meant to make people capable of understanding human rights and to enable them to take a stand for their own rights as well as those of others, so that in the long term a human rights culture may develop. Human rights education is understood as a comprehensive task which, apart from communicating knowledge, is meant to stimulate the debate on values and attitudes and to support competencies for action, which are called learning about, by way of and for human rights. From a psychological point of view, the article analyzes in how far human rights education may be implemented as a three-dimensional support of knowledge, values and skills and where, in this respect, it comes to its limits, also regarding the question of the feasibility of an attempted human rights culture. The high expectations connected with human rights education are questioned in order to get a more realistic idea of what human rights education may actually achieve.
\end{abstract}

Online publiziert: 11.05 .2012
C Die Autor(en) 2012. Dieser Artikel ist auf Springerlink.com mit Open Access verfügbar.

Dr. B. Ötsch $(\bowtie)$

Forschungs- und Studienzentrum für Pädagogik,

Universität Basel/Pädagogische Hochschule FHNW,

Riehenstrasse 154, 4058 Basel, Schweiz

E-Mail: berit.oetsch@unibas.ch 
Keywords: Cognitive development $\cdot$ Competencies $\cdot$ Human rights education $\cdot$ Psychology Values education

\section{Einleitung und Fragestellung}

Menschenrechtsbildung soll Menschen dazu befähigen, Menschenrechte zu verstehen und sich für die eigenen sowie die Rechte anderer einzusetzen, sodass langfristig eine Kultur der Menschenrechte entstehen kann. Angestoßen durch die Verabschiedung der Allgemeinen Erklärung der Menschenrechte im Jahr 1948 und dem Sozialpakt im Jahr 1966 ist die weltweite Einführung von Menschenrechtsbildung mittlerweile ein erklärtes Ziel der Vereinten Nationen, weshalb auch die Dekade der Menschenrechtsbildung (1995-2004) und das Weltaktionsprogramm für Menschenrechtsbildung (ab 2005) ausgerufen wurden. Im Entwurf zur zweiten Phase des Weltaktionsprogramms (United Nations 2010, S.4) wird Menschenrechtsbildung definiert als „any learning, education, training and information efforts aimed at building a universal culture of human rights, [...]“. Eine solche Menschenrechtskultur verlangt von der Mehrheit der Menschen eines Landes, sich mit den Menschenrechten zu identifizieren und aktiv im Sinne der Menschenrechte zu handeln (Mihr 2004).

Menschenrechtsbildung findet in verschiedenen Kontexten statt, etwa in der Schule, in der non-formalen Jugend- und Erwachsenenbildung oder der beruflichen Aus- und Weiterbildung von Professionsangehörigen (vgl. Lenhart et al. 2006). Sie wird als umfassende Aufgabe verstanden, die sich nicht nur darauf beläuft, dass Wissen vermittelt wird, sondern ebenso die Auseinandersetzung mit Werten und Einstellungen stimuliert und Handeln gefördert wird, was als Lernen über, durch und für die Menschenrechte bezeichnet wird (Arbeitsgruppe Menschenrechtsbildung im Forum Menschenrechte 2005; Deutsches Institut für Menschenrechte et al. 2005; Lohrenscheit 2002; United Nations 2003; Sommer und Stellmacher 2009; United Nations 2010). In dem Handbuch KOMPASS (Deutsches Institut für Menschenrechte et al. 2005, S. 18), welches der schulischen und außerschulischen Menschenrechtsbildungsarbeit mit Jugendlichen und jungen Erwachsenen dient und mittlerweile in vielen Sprachen vorliegt, werden die drei Bereiche wie folgt umrissen:

- Lernen über Menschenrechte (Wissen und Verstehen): Die Wahrnehmung und das Verständnis für Menschenrechtsthemen schärfen.

- Lernen durch die Menschenrechte (Einstellungen, Haltungen und Werte): Reflexion über die Menschenrechte als ethisch-normative Grundlage für das eigene Handeln.

- Lernen für die Menschenrechte (Kompetenzen und Fertigkeiten): Kompetenzen und Fertigkeiten für ein menschenrechtsbezogenes Engagement erlernen.

An dieser Stelle sei vorausgenommen, dass unter dem Begriff Kompetenzen, wie er beim Lernen für die Menschenrechte angeführt wird, im Unterschied zu anderen Definitionen (siehe dazu Weinert 2001) Schlüsselkompetenzen verstanden werden, die zum Handeln für die Menschenrechte befähigen sollen.

Diese Unterteilung der Aufgabenfelder in die drei Bereiche Wissen, Werte/Einstellungen und Handlung ist kein Spezifikum der Menschenrechtsbildung, sondern findet sich 
auch in der pädagogischen und psychologischen Fachliteratur (vgl. etwa Rost 2005 oder Sommer und Stellmacher 2009). Im Folgenden wird aus psychologischer Perspektive untersucht, inwieweit Menschenrechtsbildung als eine solche dreidimensionale Aufgabe umsetzbar ist und wo sie diesbezüglich an ihre Grenzen stößt, auch im Hinblick auf die Frage nach der Realisierbarkeit der angestrebten Menschenrechtskultur. Dabei werden folgende Fragestellungen fokussiert:

1. Inwieweit sind kognitive Voraussetzungen des Wissenserwerbs und Verstehens für die Menschenrechtsbildung von Bedeutung und setzen den Rahmen ihrer Möglichkeiten?

2. Inwieweit können Werte und Einstellungen mithilfe von Menschenrechtsbildung vermittelt werden?

3. Kann die Ausbildung von Kompetenzen, die dem Engagement für Menschenrechte dienlich sind, gefördert werden? Inwieweit kann gelernt werden, im Sinne der Menschenrechte zu handeln?

Anzumerken bleibt, dass dieser Beitrag vorwiegend Bezug auf die institutionelle Menschenrechtsbildung in der Regelschule nimmt.

\section{Lernen über Menschenrechte: Wissen und Verstehen}

Damit ein Bewusstsein für Menschenrechte entsteht, müssen diese gekannt und verstanden werden. Wissen ist demnach die notwendige Grundlage der Menschenrechtsbildung. Zum menschenrechtsrelevanten Wissen gehören das Erlernen zentraler Begriffe, wichtiger Dokumente, historischer Bezüge und der Institutionen, die für den Schutz von Menschenrechten eintreten sowie das Verstehen von (universellen) Begründungen der Menschenrechte. Die Vermittlung von Wissen ist das wichtigste Metier der Schule und somit die ,erprobteste` Domäne der drei oben genannten.

Lernen im Rahmen der Menschenrechtsbildung erfolgt vor dem Hintergrund individuell verschiedener kognitiver Voraussetzungen, weshalb nicht mit gleichen Lernergebnissen bei unterschiedlichen Personen gerechnet werden kann. Solche Unterschiede können unter Rückgriff auf psychologische Ansätze erklärt werden, was im Folgenden mit Blick auf die Relevanz für die Menschenrechtsbildung erfolgt.

Wenn auf Piagets Theorie zum logisch-kausalen Denken und auf Kohlbergs Moraltheorie Bezug genommen wird, dann geschieht das im Wissen, dass diese Ansätze wiederkehrender Kritik ausgesetzt waren und sind. Dennoch sind zentrale Grundannahmen beider Ansätze nach wie vor von Bedeutung, auch wenn sich Erweiterungen und Korrekturen innerhalb der Theorien als notwendig erwiesen haben (vgl. hierzu Becker 2011). Insbesondere die dem strukturgenetischen Ansatz zugrunde liegende Einsicht in die ontogenetisch verortete Zunahme der Differenzierungs- und Dezentrierungsfähigkeit im Denken und Urteilen scheint für die weiteren Ausführungen von Bedeutung zu sein (auf Kritik an Kohlberg hinsichtlich der Bereichsspezifität des Gerechtigkeitsurteils sei an dieser Stelle nicht eingegangen, siehe dazu Becker 2011). Kohlbergs Theorie bietet gerade für ein Verständnis des moralischen Urteilens im Rahmen der Menschenrechtsbildung auch heute noch wichtige Erklärungsansätze. 
Wissen, das im Rahmen der Menschenrechtsbildung erlernt wird, wird mit bestehenden kognitiven Strukturen (Schemata, semantischen Netzwerken, mentalen Modellen) verknüpft, wobei es zu mehr oder weniger starken Veränderungen der Strukturen kommt. Vosniadou und Brewer (1987) sprechen parallel zu den Piagetschen Begriffen Assimilation und Akkommodation von schwachen und radikalen Umstrukturierungen. Diese Anknüpfung an bereits vorhandenes Wissen bedingt, dass neue Informationen je nach Beschaffenheit des Vorwissens von verschiedenen Personen unterschiedlich integriert und interpretiert werden, ebenso von Schülern und Schülerinnen im Rahmen der Menschenrechtsbildung. Diese unterschiedliche Interpretation ist nicht nur auf generell verschiedene Erfahrungshorizonte von Personen zurückzuführen, sondern ist entwicklungspsychologisch auch durch den jeweils individuellen Entwicklungsstand der kognitiven Strukturen zu erklären. Piaget (1947/2000) hat in seiner Stadientheorie auf solche Entwicklungsschritte hingewiesen und hervorgehoben, dass das Denken in der kindlichen Entwicklung durch die zunehmende Ausdifferenzierung der kognitiven Strukturen an Abstraktheit gewinnt. Abstraktes Denken geht über das anschauliche Denken hinaus und ist gerade für ein Verständnis komplexer Begriffe und Theorien notwendig, welche auch Bestandteil der Menschenrechtsbildung sind (man denke etwa an den komplexen Begriff der Menschenwürde, s. u.). Das abstrakte Denken ist dem letzten Stadium (formal-operationale Stufe, nach Piaget etwa ab dem elften Lebensjahr) vorbehalten, welches aber nach neuerer Forschung längst nicht alle Menschen erreichen (Fend 2005; Tücke 2007). Das Erreichen dieses Stadiums ist jedoch nach Kohlberg (1976/1996) auch für das moralische (postkonventionelle) Urteilen eine notwendige Bedingung, denn die prinzipiengeleitete, postkonventionelle Moral verlangt das Verständnis abstrakter Konzepte von Gerechtigkeit und Fairness. Abhängig vom Entwicklungsstand ergeben sich demnach bestimmte Möglichkeiten und Grenzen der Verarbeitung und Beurteilung von Informationen.

Dies bedeutet selbstverständlich nicht, dass der Aufbau eines bestimmten (altersgerechten) Grundstocks an Wissen bei den meisten Menschen nicht möglich ist. Dennoch lässt sich mit Piaget auf Grenzen hinweisen: Zum einen sollte Menschenrechtsbildung am kognitiven Entwicklungsstand der Kinder und Jugendlichen ausgerichtet sein (was in vielen Unterrichtsmaterialien bereits berücksichtigt wird), zum anderen gibt es Begrenzungen nach oben, d.h. umso abstrakter die Inhalte werden, desto weniger Menschen werden diese zugänglich sein. 15-Jährige können z. B. einzelne Artikel der Allgemeinen Erklärung der Menschenrechte durchaus lernen, das Verstehen wichtiger Schlüsselbegriffe ist aber ein weitaus komplexerer Vorgang, der nicht bei jedem Jugendlichen und auch nicht bei jedem Erwachsenen vorausgesetzt werden kann.

Ein solcher Schlüsselbegriff - und zugleich der wohl zentralste im Zusammenhang mit den Menschenrechten - ist der Begriff der Menschenwürde (s. auch Abschn. 3), welcher ein schwer verständliches Konstrukt darstellt. Die Menschenwürde ist Dreh- und Angelpunkt der Menschenrechte und die Vermittlung von deren Bedeutung und Inhalten gehört sicherlich zu den schwierigsten Aufgaben in der Menschenrechtsbildung, zumal deren Begründung und inhaltliche Fundierung selbst in Expertenkreisen umstritten ist und immer wieder neu debattiert wird (vgl. Müller 2008). Der Begriff bezieht sich auf ein „hoch abstraktes philosophisches Konzept“" (Lenhart et al. 2006, S. 107); in Bezug auf die Theorie kognitiver Schemata (vgl. z. B. Edelmann 2000; Mietzel 2001; Seel 
2003) könnte man sich ein stark ausdifferenziertes Schema vorstellen, in dem der Begriff Menschenwürde mit den zugehörigen Attributen verknüpft und in den entsprechenden Kontext eingebettet ist (z. B. geschichtlicher, gesellschaftlicher oder sozialer Kontext). Das Verständnis dieses Begriffs erfordert bestimmte kognitive Voraussetzungen, d.h. die Fähigkeit zu abstraktem Denken, die nach Piaget erst im Stadium des formalen Denkens vorhanden sind. Besondere Grenzen zeigen sich bei kleineren Kindern, so ist es Grundschulkindern aufgrund der kognitiven Gegebenheiten nicht möglich, den Gehalt der Menschenwürde zu verstehen, worauf auch Lenhart et al. (2006, S. 107) hinweisen: „Menschenwürde steht zwar menschenrechtsphilosophisch als Leitidee am Anfang, aber eben nicht entwicklungslogisch beim Menschenrechtslernen von Grundschulkindern“ (dies heißt jedoch nicht, dass Grundschulkinder nicht bestimmte Grundprinzipien und Teilkonzepte, die der Idee der Menschenrechte und Menschenwürde zugrunde liegen etwa gegenseitigen Respekt - verstehen können).

Da mit den erlernten Begriffen auch Emotionen als Teil des Zusammenhangs im Gedächtnis gespeichert werden (Mietzel 2001), spielt der ,emotionale Lernkontext ${ }^{`}$ der Menschenrechtsbildung eine wichtige Rolle. Ein allzu restriktives Umfeld (hervorgerufen z.B. durch unangemessen hohen Leistungsdruck) könnte dazu führen, dass mit der Menschenrechtsbildung verbundene Begriffe in erster Linie negative Emotionen, etwa Angst, auslösen - ähnlich wie dies der Begriff Mathematik bei vielen Schülern und Schülerinnen tut ${ }^{1}$ (das bedeutet allerdings nicht, dass negative Emotionen nicht zwangsläufig auch zur Menschenrechtsbildung dazugehören, so löst der Bericht von Menschenrechtsverstößen i.d.R. negative Reaktionen wie Wut oder Empörung aus, was nicht gegen den Sinn der Menschenrechtsbildung ist). Einen ähnlichen Effekt könnte eine Menschenrechtsbildung haben, die fast ausschließlich auf negative Aspekte wie Folter oder andere Gewalttaten fokussiert, ohne auch positive Aspekte wie z. B. Errungenschaften für bzw. durch die Menschenrechte oder Freude am gemeinsamen Menschenrechtsengagement hervorzuheben.

Ein Einsatz für Menschenrechte ist wahrscheinlicher, wenn Personen mit Begriffen (oder auch Bildern), die im Zusammenhang mit Menschenrechten stehen, bestimmte Emotionen verknüpfen, z. B. Wut bei der Beobachtung von Menschenrechtsverletzungen. In diesem Empfinden von Wut kommt ein zentrales Element menschenrechtlichen Urteilens und Handelns zum Ausdruck: die Fähigkeit, sich in die Rolle und damit die Gefühle eines anderen hineinversetzen zu können, was als Empathie bezeichnet wird. In der Fähigkeit zur Empathie spiegelt sich gewissermaßen das emotionale Verständnis des Begriffs Menschenwürde wider, was bedeutet, dass die Würde des anderen durch Rollenübernahme gefühlt, somit eine Verletzung derselben als empörend erlebt wird. Das Mitfühlen mit anderen Menschen wird in der sozialen Erfahrung erlernt und ausgeformt, was sicherlich, wenn überhaupt, nur zu einem geringen Teil in der Schule möglich ist - ganz zu schweigen von der Schwierigkeit, einen bestimmten gewünschten kausalen Effekt durch Erziehung zu erzeugen. Ein weitaus größerer Teil der Gefühlserziehung wird in einem durch Bildungsmaßnahmen eher unkontrollierbaren Bereich außerhalb der Schule erfolgen, nämlich in der Familie oder dem Freundeskreis.

Abschließend sei darauf hingewiesen, dass Lernen - auch im Rahmen der Menschenrechtsbildung - kontextspezifisch ist, was bedeutet, dass Wissen, das in der Schule gelernt wird, in ,Schulschemata' abgespeichert wird, was den Transfer auf andere, außerschulische Situationen erschweren kann (vgl. Mietzel 2001). 


\section{Lernen durch Menschenrechte: Einstellungen, Haltungen und Werte}

Menschenrechtsbildung zielt auf die Veränderung von Einstellungen und Werten, sodass Menschenrechte (bzw. die diesen zugrunde liegenden Werte) als Maßstab und Grundlage für eigene Werte und Urteile angewendet werden (vgl. Deutsches Institut für Menschenrechte et al. 2005, S. 19). Auffällig ist, dass in den Materialien zur Menschenrechtsbildung selten eine Präzisierung der Begriffe Wert, Einstellung und Haltung vorgenommen wird, sodass vermutlich in der Praxis mit vagen Begriffskonzeptionen und daraus resultierenden unterschiedlichen Bedeutungsauffassungen gearbeitet wird. Im Folgenden werden einige Annahmen der den Begriffen zugrunde liegenden Konzepte, die für die Menschenrechtsbildung relevant sind, skizziert (auf den Begriff der Haltung wird dabei jedoch nicht eingegangen, da er im KOMPASS uneindeutig verwendet wird und von geringerer Relevanz für die weiteren Ausführungen ist ${ }^{2}$ ).

Werte, als explizite oder implizite Konzeptionen des Wünschenswerten (Kluckhohn 1951/1962), dienen als wichtige und höherrangige Bewertungsmaßstäbe im Leben und regulieren situationsübergreifend und objekt-unspezifisch die Wahrnehmung und Verarbeitung von Informationen (Stichwort Wert in: Häcker und Stapf 2009, S. 1090; Rokeach 1980; Tiedemann 2006).

Die Vielzahl der Werte und die Weise, in der dieselben als handlungsleitende Regulative in der menschlichen Praxis wirksam werden, trägt und verkörpert weitgehend, was ,Kultur' genannt wird (eine intersubjektive Übereinkunft hinsichtlich der Bedeutung von Werten schafft gewissermaßen, objektive“ Werte, die überindividuell als Richtschnur in einer Gemeinschaft dienen können) (vgl. Düwell et al. 2006). Werte verweisen auf Wertungen, wobei letztere sich in Form evaluativer Äußerungen zeigen, denen das Prädikat ,...ist gut‘ zugrunde liegt (vgl. Schnädelbach 2009; Tiedemann 2006). Werte werden im Alltag häufig mit Normen gleichgesetzt; allerdings stellen Normen als soziale Konventionen keine Evaluationen, sondern Präskriptionen dar, d.h. ,präskriptive Äußerungen, Vorschriften, Imperative“" (Schnädelbach 2009, S. 27). Normen sind auf kulturelle oder religiöse Traditionen zurückzuführen oder zeigen sich in Form von Gesetzen bzw. Regeln, die in einer Gemeinschaft erstellt wurden (vgl. Montada 2002). Menschenrechte verweisen somit auf Normen und Normierungen. Werte sind zentrale Bestandteile von Normen und werden in denselben realisiert, d. h. sichtbar. Auch in den Menschenrechten verankerten die Autorinnen und Autoren wichtige Werte (z. B. die von ihnen geschätzte Idee der Gleichheit), indem sie diese in ein Set von Regeln überführten, die festlegen, was als ,richtig' zu gelten hat (z. B. welche Regeln eingehalten werden müssen, damit die Gleichheit aller Menschen gewährleistet wird).

Werte liegen als Bewertungsmaßstäbe den Einstellungen zugrunde. Letztere sind im Gegensatz zu Werten stets auf ein spezifisches Objekt oder eine konkrete Situation bezogen (Seel 2003; Rokeach 1980). Diese stärkere Spezifität macht verständlich, warum die Änderung von Einstellungen im Rahmen der Menschenrechtsbildung leichter gelingen mag als die Änderung von Werten (ein Mensch, der Ausländer ablehnt, mag seine Einstellung gegenüber Menschen aus Polen ändern, wenn er einen polnischen Staatsbürger im direkten Kontakt als freundlich und zuvorkommend erlebt hat, trotz Beibehaltung des der Fremdenfeindlichkeit zugrunde liegenden Wertes). Da Einstellungen ein dynamisches System darstellen, ist eine Änderung allerdings an verschiedene Faktoren 
gebunden. Dieses System beinhaltet drei Komponenten: eine kognitive, eine affektive und eine Verhaltenskomponente (Seel 2003; Rokeach 1980), welche auch als ABC der Einstellung ,affect (feeling), behavior (intention), and cognition (thoughts)“ bezeichnet werden (Myers 1999, S. 130), d.h. ,,jede Einstellung beinhaltet Gefühle, Meinungen und Überzeugungen, Handlungen und Verhaltensweisen in Bezug auf das Einstellungsobjekt, wobei bei einer gegebenen Einstellung eine Komponente stärker als die beiden anderen ausgeprägt sein kann" (Seel 2003, S. 125). Eine Person mit einer negativen Einstellung gegenüber Ausländern glaubt z.B., diese würden Arbeitsplätze wegnehmen (Wissenskomponente), hat Angst, wenn ihr ein Fremder gegenübersteht (affektive Komponente) und wird sich vielleicht distanziert oder sogar abweisend verhalten, wenn sie von diesem nach dem Weg gefragt wird (behaviorale Komponente). Die Mehrdimensionalität von Einstellungen (affektiv, kognitiv und behavioral) rechtfertigt die Forderung nach einer Ausrichtung der Menschenrechtsbildung im schulischen Kontext als fächerübergreifendes Projekt, eingebettet in ein förderliches Schulklima (vgl. Fritzsche 2005), da dadurch neben der Wissensvermittlung auch verstärkt die Gefühls- und die Verhaltenskomponente angesprochen werden (die Schaffung eines solchen Klimas verlangt allerdings, dass das Lehrpersonal im Schulalltag die den Menschenrechten zugrunde liegenden Werte und Einstellungen überwiegend teilt und ,lebt', was nicht vorausgesetzt werden kann).

Lernprozesse, die Einstellungen und Werte betreffen, sind aufgrund der Komplexität des Gegenstands nicht beliebig steuerbar. Die verschiedenen und unterschiedlich stark ausgeprägten Komponenten von Werten und Einstellungen und das dynamische Wechselspiel zwischen diesen machen gleiche Lernprozesse mit ähnlichen und vorhersehbaren Lernergebnissen bei gleichsam allen Lernenden nahezu unmöglich. Außerdem erfolgt schulische Bildung in Konkurrenz zu wichtigen Bezugsgruppen wie der Familie oder dem Freundeskreis, weshalb ihre Wirkung deutlich schwächer ausfallen mag als häufig angenommen. Eine kausale Wirkung schulischen Unterrichts auf die Werteentwicklung und Einstellungsänderung ist daher unwahrscheinlich.

Laut Kohlbergs Stadientheorie ist zu erwarten, dass Personen, die das postkonventionelle Stadium erreicht haben, jenen Werten, die zentral für menschenrechtliches Urteilen sind, eine besondere Wichtigkeit zuschreiben. Dieses Stadium ist frühestens ab dem Alter 20 zu erwarten (Garz 1996), also nach Beendigung der Schulzeit, und wird längst nicht von allen Erwachsenen erreicht. Doch selbst, wenn in einem Bereich postkonventionelle Urteile erreicht werden, bedeutet dies noch längst nicht, dass kontextübergreifend postkonventionelle Urteile gefällt werden (zur Kritik an Kohlbergs bereichsübergreifendem Konzept siehe Beck 1996, 1999; Beck et al. 1996, 1999; Becker 2011). Auch sagt ein generelles Urteil zum Stellenwert von Menschenrechten nichts über die Beurteilung von konkreten Situationen aus. Sommer und Stellmacher (2009) konnten unter Rückgriff auf mehrere Studien zeigen, dass Personen Menschenrechte und Menschenrechtsverletzungen in Abhängigkeit vom Kontext unterschiedlich beurteilen (Principle Application $G_{a p}^{3}$ ), „demnach folgen Menschen in konkreten Situationen eher situativ bestimmten Normen und weniger universellen Normen wie Menschenrechten“ (Sommer und Stellmacher 2009, S.97).

Relevant für die Menschenrechtsbildung ist außerdem die Frage, welche Werte überhaupt zentral sind für ein Urteilen und Handeln im Sinne der Menschenrechte. Fragen nach dem Stellenwert von Werten werden u. a. in der Wertphilosophie behandelt; wel- 
chen Werten Primatstatus zukommt bzw. zukommen soll, bleibt allerdings strittig. Auch in der Menschenrechtsbildung ist nicht geklärt, welche Stellung den einzelnen Werten, die den Menschenrechten zugrunde liegen, zufällt. Zu diesen Werten werden menschliches Leben, Menschenwürde, Freiheit, Gleichheit, körperliche und seelische Unversehrtheit und soziale Sicherheit gezählt (Horster 2011). Häufig wird die Menschenwürde als zentraler, zu schützender Wert genannt (vgl. Korinek 2011). In dem bereits erwähnten Handbuch KOMPASS wird außerdem neben der Menschenwürde noch die Gleichheit als wichtigster Wert angeführt (ohne dass diese Auswahl allerdings begründet wird; so bleibt die Frage offen, warum der Gleichheit und nicht etwa der Freiheit Priorität eingeräumt wird). Während der Begriff Gleichheit einen terminalen Wert ${ }^{4}$ markiert, besteht Uneineindeutigkeit darüber, ob Menschenwürde tatsächlich zu den Werten gezählt werden kann. Vielmehr stelle der Begriff eher ein Werturteil denn einen Wert dar, denn er besage, dass dem Menschen Würde zukomme (Tiedemann 2006). ${ }^{5}$ Diese Verknüpfung zwischen Mensch und Würde verlangt nach einer Begründung. Schnädelbach (2009) verweist auf drei Begründungsversuche: einen ontologischen, einen anthropologischen und einen ethisch-normativen, wobei er den ethisch-normativen Ansatz als den einzig aussichtsreichen bezeichnet (a.a.O, S.31). Dieser steht in der kantischen Tradition und besagt, dass Würde nur einem Wesen zukommen kann, das Zweck an sich ist, also dem Menschen bzw. der Menschheit als Subjekt und Adressat der Moralität (vgl. Schnädelbach 2009). Schnädelbach (2009, S. 32) führt weiter aus: „Wie diese Kantische Idee einer ethisch-normativen Begründung der Menschenwürde heute auszubuchstabieren wäre, ist das Thema einer laufenden und weitverzweigten Diskussion [...]“ (deren Darstellung den Umfang dieses Artikels sprengen würde). Ob die Menschenwürde nun im engeren Sinne ein Wert ist oder nicht: Sie ist auf jeden Fall ein wichtiger Bezugspunkt der Menschenrechte und stellt daher ein thematisches Kernstück der Menschenrechtsbildung dar.

Der Mensch darf aufgrund der ihm zugeschriebenen Menschenwürde niemals nur als Mittel, sondern ausschließlich als Zweck an sich behandelt werden. Laut Tiedemann besitzen alle Menschen ein Bewusstsein für die Würde des anderen Menschen, da das „Bewusstsein der eigenen Würde aus einem kommunikativen Prozess hervorgeht, aus dem gleichursprünglich auch das Bewusstsein fremder Würde hervorgeht" (Tiedemann 2006, S. 101). Dies sei selbst bei den Menschen der Fall, die anderen Personen Schaden zufügen. Seine Annahme veranschaulicht Tiedemann exemplarisch anhand der Rede des SS-Reichsführers Heinrich Himmler in Posen. Himmler betonte, dass zwischen der Vernichtung von Menschen und Ratten ein Unterschied bestünde: „Im ersteren Fall werde von den Mördern die Anstrengung verlangt, keine natürlichen Gefühle gegenüber den Opfern aufkommen zu lassen, die gegenüber Ratten gar nicht bestünden, weshalb der Massenmord die ,größere Heldentat" sei“ (Tiedemann 2006, S. 100). Sogar Heinrich Himmler hatte nach Tiedemann ein Bewusstsein für die Würde des anderen zum Ausdruck gebracht, indem er von den natürlichen Gefühlen gegenüber dem anderen Menschen sprach, auch wenn er seine Handlung nicht danach ausrichtete. Das Bewusstsein für den besonderen Wert des anderen Menschen scheint bei allen Menschen vorhanden zu sein, wenngleich mehr oder weniger stark ausgeprägt. Somit läge eine Aufgabe der Menschenrechtsbildung darin, dieses schon vorhandene Bewusstsein zu explizieren und gleichzeitig zu vermitteln, warum Menschen häufig dagegen handeln. 
Die Gründe für die Zuwiderhandlung können dabei sowohl in der Persönlichkeit als auch in der Situation verortet sein. Die Bedeutung des Faktors Kontext unterstreichen mehrere Studien. Als Beispiele seien das Milgram-Experiment (Milgram 1974/2003), das Stanford-Prison-Experiment (Zimbardo 2008) oder Studien zum Rassismus (Rokeach 1980) genannt (s. a. Abschn. 4). Einen Hinweis auf den Faktor Persönlichkeit liefern z. B. die Ergebnisse von Sommer und Stellmacher (2009), die zeigen, dass Personen mit hoher Autoritarismusneigung und sozialer Dominanzorientierung Menschenrechte als weniger wichtig beurteilen (und vermutlich auch eher dagegen handeln ${ }^{6}$ ). Werte scheinen hier eine gewichtige Rolle zu spielen, so scheinen etwa für autoritäre Persönlichkeiten Ordnung und Macht wichtige Werte darzustellen (Corsini 1977). Sommer und Stellmacher (2009) unterstreichen, dass bestimmte Wertesozialisationen ein menschenrechtsbezogenes Engagement eher fördern als andere. Positive Zusammenhänge zur Unterstützung von Menschenrechten konnten sie für die Werte Universalismus und Wohlwollen ${ }^{7}$ finden, welche für ein „Überwinden von egoistischen Motiven und die Orientierung am Wohlwollen anderer Menschen“" stehen (a.a.O., S. 104), negative zu den Werten Macht und Sicherheit ${ }^{8}$ (allerdings sind die Ergebnisse bei den negativen Zusammenhängen weniger konsistent). Wie diese Werte jedoch durch Menschenrechtsbildung gefördert werden können und inwieweit dies überhaupt möglich ist, bleibt zu beantworten.

\section{Lernen für die Menschenrechte: Kompetenzen und Fertigkeiten}

Die Begriffe Kompetenz und Fertigkeit werden im KOMPASS nicht näher definiert, die aufgelisteten Fähigkeiten stellen jedoch Kompetenzen dar, die gemeinhin als klassische Schlüsselkompetenzen genannt werden, so kritisches Denken, konstruktive Konfliktbewältigung, Kommunikations- und Handlungskompetenz, kooperative Zusammenarbeit und Führungsfähigkeit.

Schlüsselkompetenzen bezeichnen kontextunabhängige Kompetenzen, genauer ,allgemeine (extrafunktionale, überfachliche, bereichsunabhängige) Strategien, Einstellungen, Fähigkeiten und Kompetenzen [...], die beim Wissenserwerb und bei der Lösung von inhaltlich sehr unterschiedlichen Problemen eingesetzt werden können“" (Reusser 2001, S. 107 f.); dazu gehören sowohl kognitive als auch sozio-emotionale Kompetenzen (Reusser 2001). Wichtigstes Merkmal der Schlüsselkompetenzen ist die Handlungskompetenz, da diese zur Bewältigung von Anforderungen von besonderer Bedeutung ist (vgl. Echterhoff 2009).

Die Beschreibung von Schlüsselkompetenzen ist - auch im Bereich der Menschenrechtsbildung - häufig ,theoretisch unscharf und mehrdeutig“ (Klieme et al. 2002, S. 204f.) und die Übertragbarkeit auf neue Situationen scheint fragwürdig (Klieme et al. 2002). Das Lehren von Schlüsselkompetenzen ist außerdem keine einfache Aufgabe, denn diese Kompetenzen sind komplexe Systeme, die auf Wissen, Überzeugungen und Handlungsschemata beruhen und sich auf ,well-organized domain-specific expertise, basic skills, generalized attitudes, and converging cognitive styles“s stützen (Weinert 2001, S. 53). Weinert weist auf die Gefahr hin, dass Schlüsselkompetenzen leicht zu schlichten Schlagwörtern werden können, sofern nicht erforscht ist, welche Strukturen und Prozesse ihnen unterliegen. Da die Forschung zu Schlüsselkompetenzen noch viele Aspekte offen 
lässt, bleibt die Frage bestehen, ob und wie solche Kompetenzen durch Erziehungsprogramme erworben werden können. So schreibt Reusser (2001, S. 108): „Was nicht nur die Alltagserfahrung lehrt, sondern auch alle wissenschaftlichen Befunde, vorab zum lernpsychologischen Problem des Transfers, deutlich machen, ist, dass es keine einfachen Lösungen zum Erwerb breit anwendbarer Schlüsselkompetenzen gibt“" Klieme et al. (2002) halten die schulische Förderung bestimmter Schlüsselkompetenzen (soziale Kompetenz, selbstreguliertes Lernen und in Maßen auch Problemlösen) zwar für möglich und sinnvoll, doch mahnen sie gleichsam zur Zurückhaltung in Bezug auf Möglichkeiten des Kompetenztransfers. Die angesprochenen Schwierigkeiten stellen auch Forderungen an die Menschenrechtsbildung: Zum einen ergibt sich die Forderung nach einer trennscharfen Definition der verwendeten Kompetenzbegriffe, zum anderen nach Absicherung der Möglichkeiten und des Erfolgs der (schulischen) Förderung und des Transfers auf andere Situationen. Das Eintreten für die Menschenrechte erfolgt selten in demselben Kontext, weshalb gewisse Schlüsselkompetenzen durchaus förderlich für menschenrechtliches Engagement sein mögen. Ob und wie diese allerdings gezielt durch schulisches Training gefördert werden können, bleibt zu klären.

Die Ausbildung bestimmter Kompetenzen durch Menschenrechtsbildung soll eine menschenrechtsorientierte Handlung bezwecken. Neben der Frage, ob Kompetenzen tatsächlich das Handeln beeinflussen oder ob das Handeln Kompetenzen formt (vgl. Sophian 1997), stellt sich die Frage, in welchem Ausmaß ein erwünschtes Handeln durch Menschenrechtsbildung überhaupt, erwirkt' werden kann.

Jede Handlung setzt voraus, dass ein Ziel festgelegt wird, welches durch ein entsprechendes Verhalten erreicht werden soll (Volpert 1974). Die Umsetzung von Handlungszielen ist ein hochkomplexer Prozess, bei dem verschiedene Faktoren eine Rolle spielen. Eine von außen erfolgende Steuerung durch Bildungsmaßnahmen in eine gewisse, erwünschte Richtung scheint daher fraglich.

In der psychologischen Forschung wird zur Erklärung des Handlungsprozesses häufig das Rubikon-Modell der Handlungsphasen verwendet. Diesseits des Rubikons ${ }^{9}$ werden die Realisierbarkeit bestimmter Wünsche sowie mögliche Handlungsergebnisse gegeneinander abgewogen (prädezisionale Phase), jenseits des Rubikons hat sich eine Zielintention gebildet und die Handlung wird eingeleitet und umgesetzt (präaktionale Phase, aktionale Phase und postaktionale Phase) (Heckhausen 1987; Kleinbeck 2006).

Während der prädezisionalen Phase erfolgt eine Abwägung von Handlungszielen, wobei es für ein menschenrechtsbezogenes Engagement wichtig wäre, dass unter den konkurrierenden Zielen eben solche ausgewählt werden, die dieses Engagement stützen (z.B. der Vorzug des Ziels der Verbesserung der Menschenrechtslage gegenüber dem Erleben von möglichst viel Freizeit). In die bewusste Auswahl an Zielen mischt sich dabei auch eine unbewusste Steuerung von Prozessen der Zielauswahl, sodass einer Person sämtliche Entscheidungsparameter schwer bewusst sein können. Außerdem werden die eigenen Ziele durch Personen aus wichtigen Bezugsgruppen beeinflusst, z. B. indem im Zuge eines Identifikationsprozesses zielrelevante Normen, Einstellungen und Verhaltensweisen von einer respektierten Gruppe oder von Menschen, die wir wertschätzen, übernommen werden (Smith et al. 2007). Neben realen Personen können aber auch fiktive Personen und Ideen aus der Literatur oder den Medien als Vorlage für die eigene Identität dienen. Für die von den Vereinten Nationen (United Nations 2010) angestrebte 
Menschenrechtskultur sollte nach Mihr (2004) eine Identifikation der Bürger mit den Menschenrechten erfolgen, was bedeutet, dass die eigenen Einstellungen und Verhaltensweisen im Sinne der Menschenrechte ausgerichtet werden und somit auch den mit den Menschenrechten verbundenen Werten wie Gleichheit oder Toleranz eine hohe Priorität unter den individuellen Werten zugesprochen wird, d.h. dass diese Werte die Festlegung von Handlungszielen mitbestimmen. Erschwerend für die Menschenrechtsbildung kommt hinzu, dass ein Verhalten im Sinne der Menschenrechte auch einen bestimmten Umgang mit Gefühlen inkludiert (z. B. Empörung, wenn andere Menschen diskriminiert werden). Die Identifikation mit den Menschenrechten ist demnach ein komplizierter Vorgang, der voraussetzt, dass der ,Vorlage“ (anderen Personen, Gruppen oder Ideen) eine hohe Wertschätzung entgegengebracht wird und ausreichend Motivation vorhanden ist, neue Einstellungen und Verhaltensweisen auszubilden bzw. alte zu korrigieren. Außerdem muss davon ausgegangen werden, dass jeder Mensch Mitglied mehrerer Bezugsgruppen ist (etwa der familiären Bezugsgruppe und der Gruppe der Mitschüler in der Schule), sich also häufig zwischen den Ansprüchen der verschiedenen Gruppen entscheiden muss.

Für ein Bestehen einer Menschenrechtskultur müsste die Prioritätensetzung zugunsten von Menschenrechtszielen bei deren Mitgliedern selbstverständlich nicht in jeder Lebenssituation erfolgen, dennoch sollten Menschenrechte bzw. deren zugrunde liegende Werte als eine Art Leitfaden im Leben dienen. Ist einmal eine Zielbindung ausgelöst, müsste das Ziel auch weiter verfolgt werden. Auch wenn eine Person den eigenen Einsatz für die Menschenrechte wünscht, bedeutet dies noch längst nicht, dass das Handlungsergebnis erfolgreich herbeigeführt wird. Inwieweit ein Handlungsplan erfolgreich ist, hängt $\mathrm{ab}$ von Personenvariablen (Habe ich genügend Wissen, um die Handlung umzusetzen? Vertraue ich in meine eigene Tüchtigkeit? Glaube ich an den Erfolg der Umsetzung?), Zielvariablen (Ist das Ziel schwierig zu erreichen? Ist es sehr komplex? Liegt die Zielerreichung in naher Zukunft oder in der Ferne?) und Situationsvariablen (Kann ich in der jeweiligen Situation die Handlung ausführen? Hindern mich z. B. Menschen aus dem Umfeld an der Ausführung der Handlung?) sowie auch der Interaktion zwischen diesen Variablen (Gibt es z. B. im Umkreis Menschen, die mein Engagement unterstützen, und besitze ich genügend soziale Kompetenz, um mich in dieser Gruppe einzubringen?).

Wie schon in Abschn. 3 angerissen, konnten Studien den starken Einfluss der Situation auf die Handlung unterstreichen - und damit die Bedeutung des personalen Faktors relativieren, auf den bisherige Konzeptionen von Menschenrechtsbildung vor allem fokussieren. Die Macht von Situationen wurde in den bekannten Experimenten von Milgram (1974/2003) oder Zimbardo (2008) deutlich. Die Situation stellt den Verhaltenskontext dar und beeinflusst somit im Zusammenspiel mit individuellen Eigenschaften die Handlung eines Menschen. Die Situation entsteht aus dem System (vgl. Zimbardo 2008), wobei das System ${ }^{10}$ aus Individuen und Organisationen besteht, ,deren Ideologie, Werte und Macht Situationen schaffen und die Rollen und Erwartungen für akzeptable Verhaltensweisen der Akteure innerhalb ihres Einflussbereichs diktieren“ (Zimbardo 2008, S. 413). Beide Forscher stellten als Abschluss ihrer Studie fest, dass es oft nicht so sehr die Wesensart des Menschen ist, die die Handlung bestimmt, sondern Merkmale der Situation, in der sich die Person befindet. So leitet Zimbardo die weiteren Ausführungen seines Buches in Analogie zum Theaterspiel mit den folgenden Worten ein (a.a. O., S. 7, Vorwort): 
Die Akteure auf der Bühne des Lebens haben in den allermeisten Fällen ein Publikum, Mitspieler, Kostüme, Requisiten und bestimmte Rollen, die sie zu spielen haben, und zumeist folgt ihr Spiel einem fertigen Skript. Wir können nicht wirklich verstehen, wie und warum ein bestimmter Akteur etwas sagt oder tut, ohne auch das Bühnenbild und sämtliche Merkmale des Verhaltenskontextes zu berücksichtigen, die seine Aufführung in vorhersehbarer Weise einschränken. [...] Es ist ebenso wichtig, neben den individuellen Dispositionen der Hauptakteure auch den Regisseur und seine Assistenten zu kennen. An dieser Stelle kommen die Systeme ins Spiel. Systemische Einflüsse auf menschliches Verhalten sind die Machtbasis, die soziale Umfelder schafft, legitimiert und aufrechterhält [...].

Da menschenrechtsbezogenes Engagement häufig in verhaltensformenden Situationen stattfindet, können sich jeder Person situative Kräfte entgegenstellen, die ein aktives Eintreten für die Menschenrechte zumindest erschweren. Menschen können zwar (mehr oder weniger gut) darin geschult werden, die Macht von Situationen zu erkennen bzw. Faktoren der Situation zu reflektieren; gleichsam verdeutlichen die Ausführungen von Milgram und Zimbardo aber, wie wichtig die Schaffung und Beibehaltung von Systemen ist, die den Einsatz für und die Gewährung von Menschenrechten ermöglichen und unterstützen.

Moralisches Handeln ist ein komplexes Geschehen und erfolgt oftmals im Rahmen vielschichtiger sozialer Beziehungen, in denen interpersonale Diskurse eine wichtige Rolle spielen und „Gemeinschaftshandlungen“ hervorrufen können (vgl. Schmid 2011, S. 265). Individuelle Eigenschaften gehen in den Prozess ein, bestimmen ihn aber weitaus geringer als häufig angenommen. „Moralisch integer wird kein in Gemeinschaft lebendes Wesen je ganz für sich allein sein können“ (a.a. O., S. 295), denn moralisches Verhalten wird stets auch zwischen Menschen in einem kooperativen, dialogischen Geschehen vereinbart (vgl. Schmid 2011). Dies gilt ebenso für menschenrechtsrelevante Kontexte.

Die Förderung von Kompetenzen und Fertigkeiten im Sinne einer Persönlichkeitsbildung kann keine Garantie für einen breiten Einsatz für die Menschenrechte liefern.

\section{Perspektiven für die Menschenrechtsbildung}

Die Erwartungen an die Ergebnisse der institutionellen Menschenrechtsbildung sind hoch. Dem stehen - wie in diesem Artikel gezeigt wurde - eingeschränkte Möglichkeiten gegenüber. Der Aspekt, der sich unter den drei genannten Bereichen (Wissen, Werte/Einstellungen, Handlung) wohl am besten für eine Förderung eignet, d. h. dessen Erwerb am ehesten geplant und kontrolliert werden kann, ist der Aufbau von Wissen über Menschenrechte. Umso bekannter die Menschenrechte sind, desto wahrscheinlicher ist es, dass sie an Bedeutung gewinnen; denn Menschenrechte, die nicht gekannt und verstanden werden, können auch nicht geachtet oder eingefordert werden (Fritzsche 2005).

Schwieriger als mit dem Lernen über Menschenrechte verhält es sich mit dem Lernen durch Menschenrechte. Die Annahme, man könne Einstellungen und Werte einem steuerbaren Lernprozess unterziehen, der nicht nur in der Schule Wirkung zeigt, sondern darüber hinaus im außerschulischen Leben, impliziert den Glauben an den von außen formbaren Charakter, der sich situationsübergreifend bewährt. Unterricht kann die Auseinandersetzung mit Werten, Einstellungen und Urteilen stimulieren, kann helfen, 
einen eigenen Standpunkt auszubilden und eine eigene Position zu beziehen bzw. eigene und fremde Ansichten zu hinterfragen. Unterricht kann einen Raum geben, sich im mitmenschlichen Diskurs mit moralischen Fragen auseinanderzusetzen, zu lernen, verschiedene Perspektiven einzunehmen sowie neben der interpersonalen auch die systemische Sichtweise zu integrieren. Er kann das Denkvermögen schulen, das soziale Miteinander fördern und den Blick für die Komplexität des Lebens und der Lebensfragen schärfen. Wohin allerdings die geistige Auseinandersetzung in der Schule einen Menschen führt, kann schwer vorausgesagt werden.

Ähnliches gilt für die Handlungskompetenz, auch hier ist nur eingeschränkt eine Förderung möglich. Abschn. 4 sollte verdeutlichen, dass die Planung und die Ausführung einer Handlung an komplexe Faktoren gebunden sind und schwer abschätzbar ist, wer in welcher Situation wie handelt. Alle Freiheitsgrade, die das Handeln regulieren, lassen sich wohl kaum festlegen, weshalb immer ein Moment des Unwägbaren bleibt. Mit Benner et al. (2010, S. 109) kann hinzugefügt werden:

Die Ausbildung eines sittlichen Charakters, wie er in antiken und modernen Begriffen der Tugend angesprochen wird, liegt außerhalb der Reichweite moralischer Urteilsbildung durch Unterricht und Handlungskompetenzsicherung im Medium öffentlicher Erziehung. Sie muss der Bewältigung der Ernstsituation des Lebens überlassen bleiben und darf, um der Sittlichkeit selbst willen, nicht unter die Kontrolle der Erziehung gebracht werden. Das hat Folgen für die begriffliche Fassung dessen, was unter moralischer Handlungskompetenz und der Konstruktion von Aufgaben zu verstehen ist, die diese zu testen sucht.

Die Forderung nach Schaffung eines Schulklimas, welches die den Menschenrechten zugrunde liegenden Werte und Ideen im Schulalltag, fühlbar' werden lässt, ist eine Forderung nach gegenseitigem Respekt und Achtung des Gegenübers, d. h. des wünschens- und erstrebenswerten Umgangs miteinander. Menschenrechtsbildung bietet die Gelegenheit, solche Fragen des Schulklimas erneut zu thematisieren sowie die Organisation von Schulwelten zu hinterfragen; gleichsam birgt die breite Umschreibung der Menschenrechtsbildung aber die Gefahr, dass eine Vielzahl an pädagogisch-psychologischen Themen mit eingeschlossen werden und dass das klare Konzept verloren geht. Zu hohe Erwartungen können schnell desillusioniert werden und so könnte auf die anfängliche Euphorie der Menschenrechtsbildung die Enttäuschung folgen. Eine Eingrenzung der Ziele der Menschenrechtsbildung würde auch das Lehrpersonal entlasten, das sich in der Lehrerausbildung wohl kaum mit dem umfassenden Konzept der Menschenrechtsbildung vertraut gemacht hat und in der jetzigen Konzeption hohem Erwartungsdruck ausgesetzt wäre.

Die durch Menschenrechtsbildung erwirkte Menschenrechtskultur soll sich durch die aktive Beteiligung der Mehrzahl der Bürger und Bürgerinnen am Einsatz für die Menschenrechte auszeichnen. Neben der Frage, ob ein solches Engagement der Mehrheit möglich ist, stellt sich die Frage, ob die Abwesenheit der breiten Mehrheit vom politischen Geschehen per se ein schlechtes Zeichen für die Demokratie und die Menschenrechte ist. Ein dauerhaftes Engagement der Mehrheit ist eher unwahrscheinlich; mit Kohlberg und Piaget konnten zumindest für das moralische Urteilen Grenzen aufgezeigt werden. Reichenbach (2000, S. 120) weist darauf hin, dass die Frage weniger zu sein scheint, ,wie viele Menschen aktiv partizipieren, als vielmehr, ob eine politische Kultur 
vorhanden ist, die diesen Namen verdient, und ob die ,passive' Mehrheit in ihren lebensweltlichen Bezügen kulturell-argumentative Strategien in jenen Belangen pflegt, die auch mit den unpolitischsten aller Kommunikationsformen , gelöst‘ werden könnten, nämlich mit Anweisung, Androhung und Befehl““. Politische Abstinenz sei nach Reichenbach nur dann beklagenswert, wenn die abstinenten Bürger und Bürgerinnen zwar eine Veränderung ihres politischen Systems wünschen, aber nicht politisch aktiv werden können oder wollen (nicht können, da z. B. negative Konsequenzen zu befürchten sind; nicht wollen, da sie etwa nicht an den Erfolg ihres eigenen Handelns glauben) (Reichenbach 2000). Ein Staat, in dem Menschenrechte gewährt und verteidigt werden, zeichnet sich wohl weniger durch die Aktivität der breiten Masse als vielmehr durch die Funktionalität des Systems als solchem aus. Ein solches System ermöglicht ein Engagement, schreibt es aber nicht vor, unterstützt die Menschen, die sich politisch engagieren möchten und versucht, die Würde aller Menschen im Staat zu schützen.

Menschenrechtsbildung mag helfen, Kinder, Jugendliche und Erwachsene für Menschenrechte einschließlich der zugehörigen Werte zu sensibilisieren. Sie mag eine Hinterfragung der eigenen Werte und Einstellungen sowie ein Engagement für die Menschenrechte bei (einigen) Personen anregen. Allerdings stößt sie schnell an ihre Grenzen, wenn die Erwartungen allzu hoch angesetzt werden, etwa wenn die Schaffung einer gesellschaftlichen Menschenrechtskultur durch Menschenrechtsbildung angestrebt wird. Eine realistische Betrachtung der Möglichkeiten, die der Menschenrechtsbildung innewohnen, kann helfen, einen Ansatz zu entwerfen, der sich in der Praxis bestmöglich umsetzen lässt und damit der Menschenrechtsbildung und letztendlich den Menschenrechten am ehesten förderlich ist.

\section{Anmerkungen}

1 Die Verknüpfung von Lerninhalten mit Emotionen konnte Bower (1995) zeigen: Emotionen, die ein Mensch beim Lernen erlebt, werden im Zusammenhang mit dem Gelernten im Gedächtnis gespeichert.

2 Es wird im KOMPASS nicht deutlich, ob der Begriff Haltung entsprechend dem Duden (1985) synonym für den Begriff Einstellung verwendet wird oder ob Bezug auf die psychologische Definition der Werthaltung genommen wird. Von Werthaltungen wird gesprochen, wenn Werte der Handlungsorientierung dienen, und dieses über viele spezifische Situationen, Personen und Gegenstände hinweg (Spektrum Akademischer Verlag 2012), d.h. sie werden auch ,als Handlungsschemata verstanden, die durch abstrakte Begriffe (z. B. Gerechtigkeit, Wohlfahrt) repräsentiert werden und das Handeln im sozialen Feld, zumindest aber die überdauernden Einstellungen einer Person gegenüber Dingen und Personen maßgeblich beeinflussen“ (Seel 2003, S. 127).

3 Der Begriff Principle Application Gap beschreibt die beobachtete Verzerrung bei der Anwendung von (Menschenrechts-)Normen. Die Wichtigkeit der Verwirklichung von bestimmten Rechten wird meist höher eingeschätzt als die Verurteilung der Verletzung dieser Rechte in konkreten Situationen (Sommer und Stellmacher 2009).

4 Rokeach (1980) unterteilt Werte in instrumentelle und terminale Werte. Er nennt 18 instrumentelle Werte (diese beziehen sich auf Verhaltensweisen, die eine Person unabhängig von der Situation und dem Objekt bevorzugt und die Mittel zum Zweck sind, z. B. Ehrlichkeit) und 18 terminale Werte (diese sind auf Endzustände der Existenz gerichtet, etwa die Erreichung von Frieden). 
5 Der Bewertungsmaßstab, auf den sich das Werturteil Dem Menschen kommt Würde zu stützt, ist laut Tiedemann (2006) die Willensfreiheit als absoluter Wert, d. h. Menschenwürde ist der Begriff einer Werteigenschaft, die Menschen aufgrund ihres freien Willens zukommt.

6 An dieser Stelle sei darauf hingewiesen, dass der Zusammenhang zwischen Einstellung und Verhalten nach wie vor ungeklärt ist (vgl. Heidbrink 2008; Myers 1999; Seel 2003).

7 Universalismus enthält Werte wie Frieden, Gleichheit, Toleranz und soziale Gerechtigkeit, die sich auf das Wohlergehen von Personen und sozialen Gruppen generell beziehen; Wohlwollen beinhaltet Werte wie hilfreich, ehrlich oder verantwortlich sein, die sich auf das Wohlergehen von Personen der eigenen Gruppe oder des eigenen Freundeskreises beziehen (Sommer und Stellmacher 2009).

8 Sicherheit beinhaltet Werte wie soziale Ordnung oder nationale Sicherheit, Macht hingegen Werte wie Autorität, Wohlstand oder soziale Anerkennung. Bei diesen Werten stehen die Durchsetzung individueller Interessen oder der Interessen der eigenen Gruppe im Vordergrund (Sommer und Stellmacher 2009).

9 Der Begriff Rubikon nimmt Bezug auf die Überschreitung des Rubikons durch Julius Cäsar 49 v. Chr. Mit der Überquerung des Flusses und dem damit verbundenen Verlassen seines Amtsbereichs trat Cäsar einen Bürgerkrieg los.

10 Dem Begriff System unterliegen je nach Perspektive und Fachdisziplin verschiedene Definitionen und Ausformulierungen, die hier in ihrer Bandbreite nicht diskutiert werden (können).

Open Access: Dieser Artikel unterliegt den Bedingungen der Creative Commons Attribution License. Dadurch sind die Nutzung, Verteilung und Reproduktion erlaubt, sofern der/die Originalautor/en und die Quelle angegeben sind.

\section{Literatur}

Arbeitsgruppe Menschenrechtsbildung im Forum Menschenrechte (2005). Standards der Menschenrechtsbildung in Schulen. http:/www.unesco.de/fileadmin/medien/Dokumente/Wissenschaft/Bildungsstandards_MRB.pdf. Zugegriffen: 2. Feb. 2012.

Beck, K. (1996). „Berufsmoral“ und „Betriebsmoral“. Didaktische Konzeptualisierungsprobleme einer berufsqualifizierenden Moralerziehung. In K. Beck (Hrsg.), Berufserziehung im Umbruch. Didaktische Herausforderungen und Ansätze zu ihrer Bewältigung (S. 125-142). Weinheim: Deutscher Studien Verlag.

Beck, K. (1999). Die Entwicklung moralischer Urteilskompetenz in der kaufmännischen Erstausbildung - Zur Analyse der Segmentierungshypothese. In P. F. E. Sloane, R. Bader \& G. A. Straka (Hrsg.), Lehren und Lernen in der beruflichen Aus- und Weiterbildung. Ergebnisse der Herbsttagung 1998 (S.35-41). Opladen: Leske + Budrich.

Beck, K., Brütting, B., Lüdecke-Plümer, S., Minnameier, G., Schirmer, U., \& Schmid, S. N. (1996). Zur Entwicklung moralischer Urteilskompetenz in der kaufmännischen Erstausbildung. Empirische Befunde und praktische Probleme. In K. Beck \& H. Heid (Hrsg.), Beiheft 13 zur ZBW „Lehr-Lern-Prozesse in der kaufmännischen Erstausbildung. Wissenserwerb, Motivierungsgeschehen und Handlungskompetenzen " (S. 187-206). Stuttgart: Steiner.

Beck, K., Heinrichs, K., Minnameier, G., \& Parche-Kawik, K. (1999). Homogeneity of moral judgment? - apprentices solving business conflicts. Journal of Moral Education, 28(4), 429-443.

Becker, G. (2011). Kohlberg und seine Kritiker. Die Aktualität von Kohlbergs Moralpsychologie. Eine systematische und forschungsgeschichtliche Analyse. Wiesbaden: VS Verlag für Sozialwissenschaften. 
Benner, D., Nikolova, R., \& Swiderski, J. (2010). Die Entwicklung moralischer Kompetenzen als Aufgabe des Ethik-Unterrichts an öffentlichen Schulen. Zur Konzeption des DFG-Projekts ETiK. http://www.topologik.net/Dietrich_Benner_Roumiana_Nikolova_Jana_Swiderski_ Topologik_7.pdf. Zugegriffen: 2. Feb. 2012.

Bower, G. H. (1995). Emotion and social judgments. www.genesis.users.gxn.net/Emotion\%20 and\%20social\%20judgments.doc. Zugegriffen: 2. Feb. 2012.

Corsini, R. J. (1977). Current personality theories. Itasca: Peacock.

Deutsches Institut für Menschenrechte, Bundeszentrale für Politische Bildung \& Europarat (2005). KOMPASS - Handbuch zur Menschenrechtsbildung für die schulische und außerschulische Bildungsarbeit. Bonn: Bundeszentrale für politische Bildung.

Duden (1985). Das Bedeutungswörterbuch. Mannheim: Dudenverlag.

Düwell, M., Hübenthal, C., \& Werner, M. H. (2006). Handbuch Ethik (2., akt. und erw. Aufl.). Stuttgart: Metzler.

Echterhoff, W. (2009). Schlüsselqualifikation. In H. O. Häcker \& K.-H. Stapf (Hrsg.), Dorsch. Psychologisches Wörterbuch (S. 882). Bern: Huber.

Edelmann, W. (2000). Lernpsychologie (6., vollst. überarb. Aufl.). Weinheim: Beltz PVU.

Fend, H. (2005). Entwicklungspsychologie des Jugendalters (3.Aufl.). Wiesbaden: VS Verlag für Sozialwissenschaften.

Fritzsche, K. P. (2005). Die Macht der Menschenrechte und die Schlüsselrolle der Menschenrechtsbildung. Der Bürger im Staat, 55(1/2), 64-70.

Garz, D. (1996). Lawrence Kohlberg. Zur Einführung. Hamburg: Junius.

Häcker, H. O., \& Stapf, K.-H. (Hrsg.) (2009). Dorsch. Psychologisches Wörterbuch (15., überarb. und erw. Aufl.). Bern: Huber.

Heckhausen, H. (1987). Perspektiven einer Psychologie des Wollens. In H. Heckhausen, P. M. Gollwitzer \& F. E. Weinert (Hrsg.), Jenseits des Rubikon: der Wille in den Humanwissenschaften (S. 121-142). Berlin: Springer.

Heidbrink, H. (2008). Einführung in die Moralpsychologie. Weinheim: Beltz.

Horster, D. (2011). Zukunft der Menschenrecht[e] - Anspruch und Wirklichkeit. http://detlef.horster.phil.uni-hannover.de/texte/menschenrechte1.pdf. Zugegriffen: 2. Feb. 2012.

Kleinbeck, U. (2006). Handlungsziele. In J. Heckhausen \& H. Heckhausen (Hrsg.), Motivation und Handeln (S. 255-276). Heidelberg: Springer.

Klieme, E., Artelt, C., \& Stanat, P. (2002). Fächerübergreifende Kompetenzen: Konzepte und Indikatoren. In F. E. Weinert (Hrsg.), Leistungsmessungen in Schulen (S. 203-218). Weinheim: Beltz.

Kluckhohn, C. (1951/1962). Values and value-orientations in the theory of action: An exploration in definition and classification. In T. Parsons \& E. A. Shils (Hrsg.), Toward a general theory of action (S. 388-433). Cambridge: Harvard University Press.

Kohlberg, L. (1976/1996). Moralstufen und Moralerwerb: der kognitiv-entwicklungstheoretische Ansatz. In W. Althof, G. Noam \& F. Oser (Hrsg.), Lawrence Kohlberg. Die Psychologie der Moralentwicklung (S. 123-174). Frankfurt a. M.: Suhrkamp.

Korinek, K. (2011). Der Schutz der Menschenwürde im Verfassungsrecht und im internationalen Recht. In F. Harrer, H. Honsell \& P. Mader (Hrsg.), Gedächtnisschrift für Theo Mayer-Maly (S. 257-268). Wien: Springer.

Lenhart, V., Druba, V., \& Batarilo, K. (2006). Pädagogik der Menschenrechte (2., überarb. und akt. Aufl.). Wiesbaden: VS Verlag für Sozialwissenschaften.

Lohrenscheit, C. (2002). International approaches in human rights education. International Review of Education, 48(3-4), 173-185.

Mietzel, G. (2001). Pädagogische Psychologie des Lernens und Lehrens. Göttingen: Hogrefe.

Mihr, A. (2004). Demokratie, Menschenrechtskultur und Menschenrechtsbildung in Deutschland. In C. Mahler \& A. Mihr (Hrsg.), Menschenrechtsbildung. Bilanz und Perspektiven (S.219231). Wiesbaden: VS Verlag für Sozialwissenschaften.

Milgram, S. (1974/2003). Das Milgram-Experiment (13. Aufl.). Reinbek: Rowohlt. 
Montada, L. (2002). Moralische Entwicklung und moralische Sozialisation. In R. Oerter \& L. Montada (Hrsg.), Entwicklungspsychologie (S. 619-647). Weinheim: Beltz.

Müller, J. (2008). Ein Phantombild der Menschenwürde: Begründungstheoretische Überlegungen zum Zusammenhang von Menschenrechten und Menschenwürde. In G. Brudermüller \& K. Seelmann (Hrsg.), Menschenwürde. Begründungen, Konturen, Geschichte (S.117-147). Würzburg: Königshausen \& Neumann.

Myers, D. G. (1999). Social psychology (6.Aufl.). Boston: McGraw-Hill College.

Piaget, J. (1947/2000). Die Psychologie der Intelligenz (10. Aufl.). Stuttgart: Klett-Cotta.

Reichenbach, R. (2000). Die Ironie der politischen Bildung - Ironie als Ziel politischer Bildung. In R. Reichenbach \& F. Oser (Hrsg.), Zwischen Pathos und Ernüchterung. Zur Lage der Politischen Bildung in der Schweiz / Entre pathos et désillusion. La situation de la formation politique en Suisse (S. 118-130). Freiburg, CH: Universitätsverlag.

Reusser, K. (2001). Unterricht zwischen Wissensvermittlung und Lernen lernen. Alte Sackgassen und neue Wege in der Bearbeitung eines pädagogischen Jahrhundertproblems. In C. Finkbeiner \& G. W. Schnaitmann (Hrsg.), Lehren und Lernen im Kontext empirischer Forschung und Fachdidaktik (S. 106-140). Donauwörth: Auer.

Rokeach, M. (1980). Beliefs, attitudes and values. A theory of organization and change. San Francisco: Jossey-Bass.

Rost, J. (2005). Messung von Kompetenzen Globalen Lernens. Zeitschrift für internationale Bildungsforschung und Entwicklungspädagogik, 28(2), 14-18.

Schmid, H. B. (2011). Moralische Integrität. Kritik eines Konstrukts. Berlin: Suhrkamp.

Schnädelbach, H. (2009). Werte und Würde. In C. Thies (Hrsg.), Der Wert der Menschenwürde (S. 21-32). Paderborn: Schöningh.

Seel, N. (2003). Psychologie des Lernens. Lehrbuch für Pädagogen und Psychologen (2., akt. und erw. Aufl). München: Reinhardt.

Smith, E. E., Nolen-Hoeksema, S., Fredrickson, B. L., \& Loftus, G. R. (2007). Atkinsons und Hilgards Einführung in die Psychologie (14.Aufl.). Berlin: Spektrum.

Sommer, G., \& Stellmacher, J. (2009). Menschenrechte und Menschenrechtsbildung. Eine psychologische Bestandsaufnahme. Wiesbaden: Verlag für Sozialwissenschaften.

Sophian, C. (1997). Beyond competence. The significance of performance for conceptual development. Cognitive Development, 12, 281-303.

Spektrum Akademischer Verlag (2012). Online-Lexikon der Psychologie. Stichwort Werthaltung. http://www.wissenschaft-online.de/abo/lexikon/psycho/16786. Zugegriffen: 2. Feb. 2012.

Tiedemann, P. (2006). Was ist Menschenwürde? Eine Einführung. Darmstadt: WBG.

Tücke, M. (2007). Entwicklungspsychologie des Kindes- und Jugendalters für (zukünftige) Lehrer (3. Aufl.). Berlin: LIT.

United Nations (2003). ABC, teaching human rights. Practical activities for primary and secondary schools. http://www.hrea.org/erc/Library/display_doc.php?url=http\%3A\%2F\%2Fwww.hrea. org\%2Ferc\%2FLibrary\%2FABC.pdf\&external=N. Zugegriffen: 2. Feb. 2012.

United Nations (2010). Draft plan of action for the second phase (2010-2014) of the World Programme for Human Rights Education. Note by the United Nations High Commissioner for Human Rights. http://www2.ohchr.org/english/bodies/hrcouncil/docs/15session/A. HRC.15.28 en.pdf. Zugegriffen: 2. Feb. 2012.

Volpert, W. (1974). Handlungsstrukturanalyse als Beitrag zur Qualifikationsforschung. Köln: Pahl-Rugenstein.

Vosniadou, S., \& Brewer, W. F. (1987). Theories of knowledge restructuring in development. Review of Educational Research, 57(1), 51-67.

Weinert, F. E. (2001). Concept of competence: A conceptual clarification. In D. S. Rychen \& L. H. Salganik (Hrsg.), Defining and selecting key competencies (S. 45-65). Seattle: Hogrefe \& Huber.

Zimbardo, P. G. (2008). Der Luzifer-Effekt. Die Macht der Umstände und die Psychologie des Bösen. Heidelberg: Spektrum. 